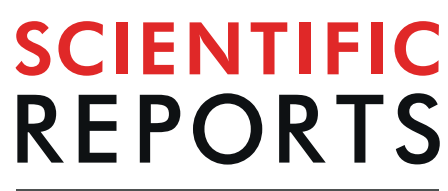

natureresearch

\title{
OPEN Human mesenchymal stem cell sheets in xeno-free media for possible allogenic applications
}

Received: 10 January 2019

Accepted: 11 September 2019

Published online: 08 October 2019
Kyungsook Kim ${ }^{1}$, Sophia Bou-Ghannam ${ }^{1,2}$, Hallie Thorp ${ }^{1,2}$, David W. Grainger ${ }^{1,2}$ \& Teruo Okano ${ }^{1,3}$

Cell-based therapies are increasingly focused on allogeneic stem cell sources because of several advantages in eliminating donor variability (e.g., aging and disease pathophysiology) affecting stem cell quality and in cell-banked sourcing of healthy donors to enable "off-the-shelf" products. However, allogeneic cell therapy is limited by host patient immunologic competence and inconsistent performance due to cell delivery methods. To address allogeneic cell therapy limitations, this study developed a new allogeneic stem cell sheet using human umbilical cord mesenchymal stem cells (hUCMSC) that present low antigenicity (i.e., major histocompatibility complex, MHC). Optimal conditions including cell density, passage number, and culture time were examined to fabricate reliable hUCMSC sheets. MHC II antigens correlated to alloimmune rejection were barely expressed in hUC-MSC sheets compared to other comparator MSC sheets (hBMSC and hADSC). hUC-MSC sheets easily graft spontaneously onto subcutaneous tissue in immune-deficient mice within 10 minutes of placement. No sutures are required to secure sheets to tissue because sheet extracellular matrix (ECM) actively facilitates cell-target tissue adhesion. At 10 days post-transplantation, hUC-MSC sheets remain on ectopic target tissue sites and exhibit new blood vessel formation. Furthermore, implanted hUC-MSC sheets secrete human HGF continuously to the murine target tissue. hUC-MSC sheets described here should provide new insights for improving allogenic cell-based therapies.

Mesenchymal stem cells have been an interest for allogeneic cell-based therapies for decades ${ }^{1,2}$. Nearly 500 clinical trials using mesenchymal stem cell (MSC) therapies (http://www.clinicaltrial.gov/) have treated over 2000 patients to date ${ }^{2}$. Many of these involve intravenous infusions of either autologous or allogenic MSCs as cell suspensions. Therapeutic benefits from any of these trials is arguably marginal to date, despite reasonable preclinical evidence. Consensus on mechanisms for MSC cell therapy does not currently exist. Nonetheless, several hypotheses have been forward to explain observed MSC clinical benefits ${ }^{3}$, specifically, their intrinsic ability to (1) differentiate into diverse and distinct cell lineages, (2) produce an array of soluble bioactive factors central to cell maintenance, survival and proliferation, (3) modulate host immune responses, and (4) migrate as recruited to sites of injury to mitigate damage and promote healing (i.e., homing) ${ }^{2}$. In certain reported cases, MSCs seemingly avoid allogeneic rejection in humans and in animal models ${ }^{4-8}$. For these reasons, MSCs have frequently been used to treat various diseases such as myocardial infarcts, graft-versus-host disease, Crohn's Disease, cartilage and meniscus repair, stroke, and spinal cord injury ${ }^{2,9-11}$. This produces realistic possibilities for pioneering allogeneic cell therapies that, as off-the-shelf products, might someday side-step the unfavorable costs and development disincentives associated with autologous stem cell treatment paradigms ${ }^{12}$.

More practically, allogeneic cell sources must be able to demonstrate their reliable capabilities to elicit meaningful therapies under standard immunologic competence in host patient allogeneic tissues. This includes reliable cell homing to and fractional dose engraftment or retention for sufficient duration at the tissue site of therapeutic interest ${ }^{13}$. Current estimates are that less than $3 \%$ of injected stem cells are retained in damaged myocardium 3 days post-injection following ischemic injury ${ }^{14}$. Additionally, most administered cells that engraft into target

${ }^{1}$ Cell Sheet Tissue Engineering Center (CSTEC), Department of Pharmaceutics and Pharmaceutical Chemistry, Health Sciences, University of Utah, 30 South 2000 East, Salt Lake City, Utah, 84112, USA. 'Department of Biomedical Engineering, University of Utah, 36 SWasatch Dr, Salt Lake City, Utah, 84112, USA. ${ }^{3}$ Institute of Advanced Biomedical Engineering and Science, Tokyo Women's Medical University, 8-1 Kawada-cho, Shinjuku-ku, Tokyo, 162-8666, Japan. Correspondence and requests for materials should be addressed to K.K. (email: kyungsook.kim@utah.edu) orT.O. (email: teruo.okano@utah.edu) 


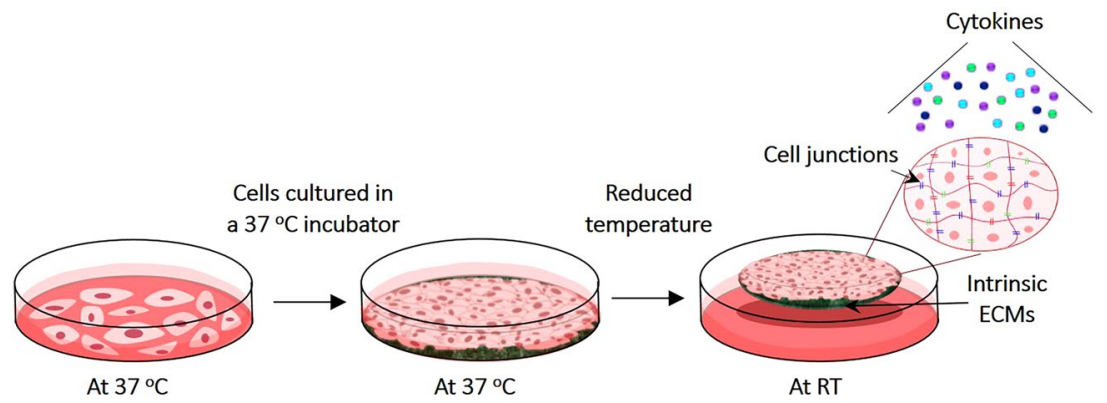

Figure 1. Cell sheet experimental protocol. Human umbilical cord stem cells (hUC-MSCs) were seeded on temperature responsive cell culture dishes (TRCD) and cultured to confluence in a $37^{\circ} \mathrm{C}$ cell culture incubator. Cultured cells were detached from TRCDs as intact cell sheets within $20 \mathrm{~min}$ at room temperature (RT), preserving their functional structures such as extracellular matrixes (ECMs) and cell-cell-junctions and cytokine secretion ability without using proteolytic enzyme treatments.

tissue will die within the first few weeks ${ }^{15}$. Effective translation of MSC therapies is currently hindered by the clinical inability to target these therapeutic cells to tissues of interest with reasonable efficiency and significant engraftment and retention. Conventional MSC therapies are injectable cell suspensions, often derived from culture-adherent cells harvested from culture plastics using proteolytic enzymes. Proteolyzed, dissociated cells require substantial time to recover from harvest, suspension and loss of cell-cell junctions, associated matrix and cell receptors. MSCs maintained in two-dimensional (2D) culture systems are shown to gradually lose intrinsic proliferative potential, colony-forming efficiency, and differentiation capacity over time ${ }^{16-18}$. Additionally, MSC homing to target tissue areas are compromised because intrinsic MSC adhesion components and mechanisms are damaged by proteolytic enzyme treatment ${ }^{19,20}$. Integrating healing physiology and regenerative potential is reduced by low cell retention and engraftment into target tissues and organs, a key factor in successful cell therapy $^{21}$.

Human umbilical cord-derived MSCs (hUC-MSCs) used in this study represent a promising allogeneic cell source for stem cell therapy among diverse MSC types, with increasing clinical evidence ${ }^{22-25}$. hUC-MSCs exhibit low HLA expression and higher paracrine effects compared to human bone marrow stem cells (hBM-MSC) ${ }^{22,26,27}$. Furthermore, intravenously infused allogenic hUC-MSC treatments induced no adverse host immune responses and produced clinically significant improvements in patients either with heart failure, with spinal cord, or with multiple sclerosis ${ }^{22-25}$. Despite these optimistic early results, cell delivery and engraftment must be improved because few injected cells reach target tissue sites with sufficiently long retention or viability to enact reliable therapeutic effects.

Okano and colleagues previously developed a versatile cell delivery method exploiting new cell culture capabilities from temperature-responsive cell culture dishes (TRCD) ${ }^{28,29}$. These polymer-grafted tissue culture surfaces release cultured cells as confluent living sheets in response to small changes in culture temperature, notably without enzymes. Recovered cell sheets completely retain native forms, confluent phenotypes and organization, cell-cell communication, intact extracellular matrix (ECM) and tissue-like behaviors (Fig. 1) ${ }^{20,30,31}$. Moreover, intact ECM decorating cell sheets serves as a natural tissue adhesive, eliminating needs to suture. This allows cell sheets to rapidly and spontaneously attach to target tissues. This feature increases their engraftment efficiency ${ }^{30,32,33}$. Using this cell sheet technology and autologous patient cell sources, Okano and colleagues have treated several human diseases in clinical trials ${ }^{33-43}$. Motivated by the compelling need to develop allogeneic cell sheets for more widespread use of such therapies, MSC sheets have been proposed ${ }^{30,44,45}$. This study aimed to develop and characterize a potential allogeneically acceptable hUC-MSC sheet. Towards this goal, baseline criteria to prepare hUC-MSC sheets were optimized. hUC-MSC sheets fabricated using this strategy were evaluated both structurally and functionally.

\section{Results}

hUC-MSC sheet preparation by varying initial cell seeding and passage numbers. hUC-MSCs were cultured on flasks and sub-cultured using trypsin every 5 days from passages 4 to 12 (Table 1). Cells were proliferated 16-20 times from initial cell seeding numbers between passages 4-8 during sub-culture. However, cell proliferation rates dramatically decrease from passage 9. Cell numbers were 14, 10.9, 7.5, and 3.1-fold increased from initial cell seeding numbers at passage $9,10,11$, and 12 , respectively. Cells in passage 10 required one day more to reach confluence and yield cell sheets than cells in passages $4-8$ at the same seeding density (Fig. 2a,b). Cells in passage 12 exhibited heterogeneously cultured morphologies, lost contact inhibition, and clumped into multi-layered aggregates rather than consistent monolayers (Fig. 2a). When culture temperature was reduced to RT, cells in passage 12 detached from TRCD, but not as sheets, as recovered from passages 4-10 (Fig. 2b). Passage 12 cells were not able to form stable sheets due to reduced cell proliferation rates and inadequate cell-cell junction formation after increased passaging (Fig. S1 and Table 1). Cells should therefore be used from passages 4 to 8 to produce consistent cell sheet quality.

Initially seeded cell numbers of $5 \times 10^{4}, 1 \times 10^{5}$, and $2 \times 10^{5}$ cells/dish in passage 6 reached confluence at 6,5 , and 4 days, respectively (Fig. 3a,b), reliably producing hUC-MSC sheets (Fig. 3c). Cell sheets from all different initial cell number groups spontaneously detached from TRCDs without temperature change (i.e., at $37^{\circ} \mathrm{C}$ ) at 5 , 


\begin{tabular}{|l|l|l|l|l|}
\hline & $\begin{array}{l}\text { Cell } \\
\text { viability }\end{array}$ & $\begin{array}{l}\text { Initial cell } \\
\text { seeding density }\end{array}$ & $\begin{array}{l}\text { Expanded cell } \\
\text { density }\end{array}$ & $\begin{array}{l}\text { Fold } \\
\text { increase }\end{array}$ \\
\hline Passage 4 & $96.0 \%$ & $4.0 \times 10^{3} / \mathrm{cm}^{2}$ & $6.5 \times 10^{4} / \mathrm{cm}^{2}$ & 16.3 \\
\hline Passage 5 & $96.0 \%$ & $3.5 \times 10^{3} / \mathrm{cm}^{2}$ & $6.7 \times 10^{4} / \mathrm{cm}^{2}$ & 19.0 \\
\hline Passage 6 & $97.5 \%$ & $3.2 \times 10^{3} / \mathrm{cm}^{2}$ & $5.5 \times 10^{4} / \mathrm{cm}^{2}$ & 17.2 \\
\hline Passage 7 & $96.2 \%$ & $3.2 \times 10^{3} / \mathrm{cm}^{2}$ & $5.5 \times 10^{4} / \mathrm{cm}^{2}$ & 17.3 \\
\hline Passage 8 & $96.7 \%$ & $3.2 \times 10^{3} / \mathrm{cm}^{2}$ & $6.6 \times 10^{4} / \mathrm{cm}^{2}$ & 20.7 \\
\hline Passage 9 & $98.5 \%$ & $3.2 \times 10^{3} / \mathrm{cm}^{2}$ & $4.5 \times 10^{4} / \mathrm{cm}^{2}$ & 14.0 \\
\hline Passage 10 & $97.6 \%$ & $3.2 \times 10^{3} / \mathrm{cm}^{2}$ & $3.5 \times 10^{4} / \mathrm{cm}^{2}$ & 10.9 \\
\hline Passage 11 & $97.4 \%$ & $3.2 \times 10^{3} / \mathrm{cm}^{2}$ & $2.4 \times 10^{4} / \mathrm{cm}^{2}$ & 7.5 \\
\hline Passage 12 & $97.4 \%$ & $3.2 \times 10^{3} / \mathrm{cm}^{2}$ & $1.0 \times 10^{4} / \mathrm{cm}^{2}$ & 3.1 \\
\hline
\end{tabular}

Table 1. Growth rates for hUC-MSCs in passages 4-12.

(a)

(b)
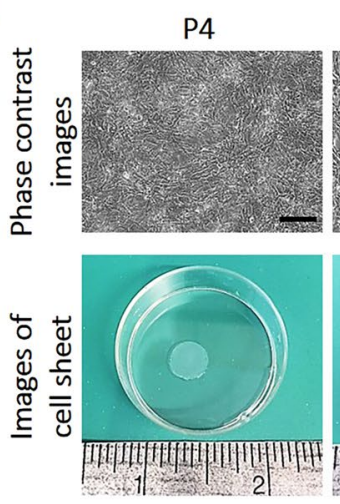

P6
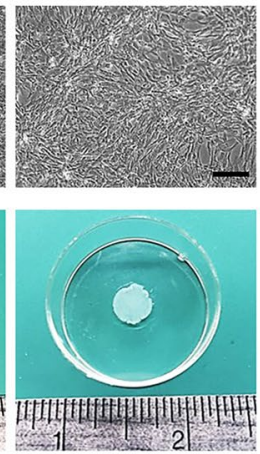

P8
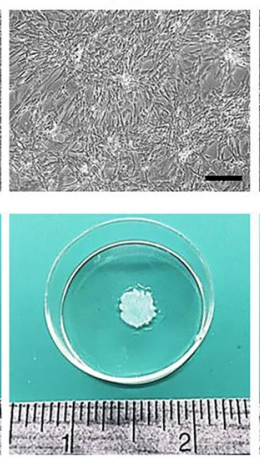

P10
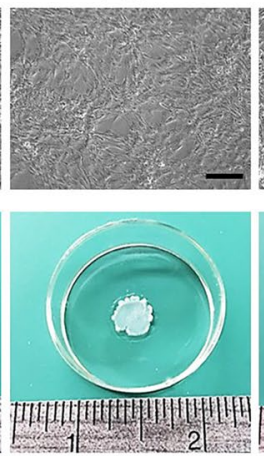

P12
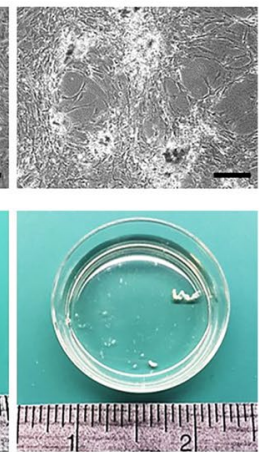

Figure 2. Passaging influence. hUC-MSC sheet morphological observations using cell passages 4, 6, 8, 10 and 12 seeded at $3.2-4 \times 10^{3}$ cells $/ \mathrm{cm}^{2}$. (a) Morphology of passage $4,6,8,10$ and 12 cells observed using phasecontrast microscopy before sheet detachment. (b) Successful fabrication of hUC-MSC sheets using passage 4, 6, 8 and 10 cells. In contrast, passage 12 cells detached as non-contiguous disconnected cellular structures. Scale bars $=100 \mu \mathrm{m}$.

6 , and 7 days in the $2 \times 10^{5}, 1 \times 10^{5}$, and $5 \times 10^{4}$ seeded cells/dish groups, respectively (Fig. $3 \mathrm{c}$ ) once cells were over-confluent. One day prior to cell confluence, adherent cells were less than $70-80 \%$ confluent and detached as partially broken sheet fragments when temperature was reduced to RT due to insufficient cell density. Recovery of hUC-MSC sheets either one day before or one day after cells reaching confluence on TRCDs was not possible (Fig. 3c). These results indicate that cell sheets must be prepared carefully and recovered at the precise time-point when cells are neither under- or over-confluent in order to detach the cells as a thermally recovered sheet.

hUC-MSC multi-potency and stem cell surface marker characterization. To qualify the cell source, hUC-MSC differentiation potential and stem cell phenotype were characterized for passages 6, 8, 10, and 12 . Positive osteogenic and adipogenic differentiation were both observed in induced hUC-MSC cultures from passage 6, 8, 10, and 12 via Alizarin Red and Oil Red O staining, respectively (Fig. 4a). Positive osteogenic and adipogenic differentiation declined from passage 6 through passage 12, with passage 12 cultures showing minimal differentiation. hUC-MSCs expressed a surface phenotype consistent with mesenchymal stem cells from passage 6 to 10, showing positive expression of CD73, CD105, and CD90. Passage 12 cells showed lower positive expression of CD73, CD105 and CD90, compared to passage 6-10 cells (Fig. 4b). hUC-MSCs in all passage numbers were negative for HLA-DR, DP, and DQ, CD45, and CD31 by qPCR. Therefore, hUC-MSC passages 6 should be appropriate to prepare hUC-MSC sheets with higher multi-potency while maintaining stem cell phenotypes.

Allogeneic immune response of hUC-MSC sheet. To verify MHC II antigen expression of hUC-MSC sheet, quantitative RT-PCR analysis was performed to measure HLA-DR, DP, and DQ gene expression levels of hUC-MSC sheets, compared to other types of MSC sheets (hBMSC and hADSC sheets) at passage 6. HLA-DR and DQ gene expression levels in hUC-MSC sheets were not detectable while hADSC and BMSC sheets clearly exhibited HLA-DR and DQ gene expression. HLA-DP gene expression levels in hUC-MSC sheets were significantly lower than other hBMSC and hADSC sheets (Fig. 4c). These data demonstrate that hUC-MSC sheets lack or bear low MHC II antigens compared to other types of human MSC sheets.

Structural analysis of hUC-MSC sheets. Passage 6 cells were cultured on TRCD for 4 days and resulting cell sheets were recovered from TRCD using temperature reduction to RT. The cell sheet was stained for fibronectin, laminin, $\beta$-catenin, and CD44 to verify hUC-MSC sheet retention of functional structures and stem cell 
(a)
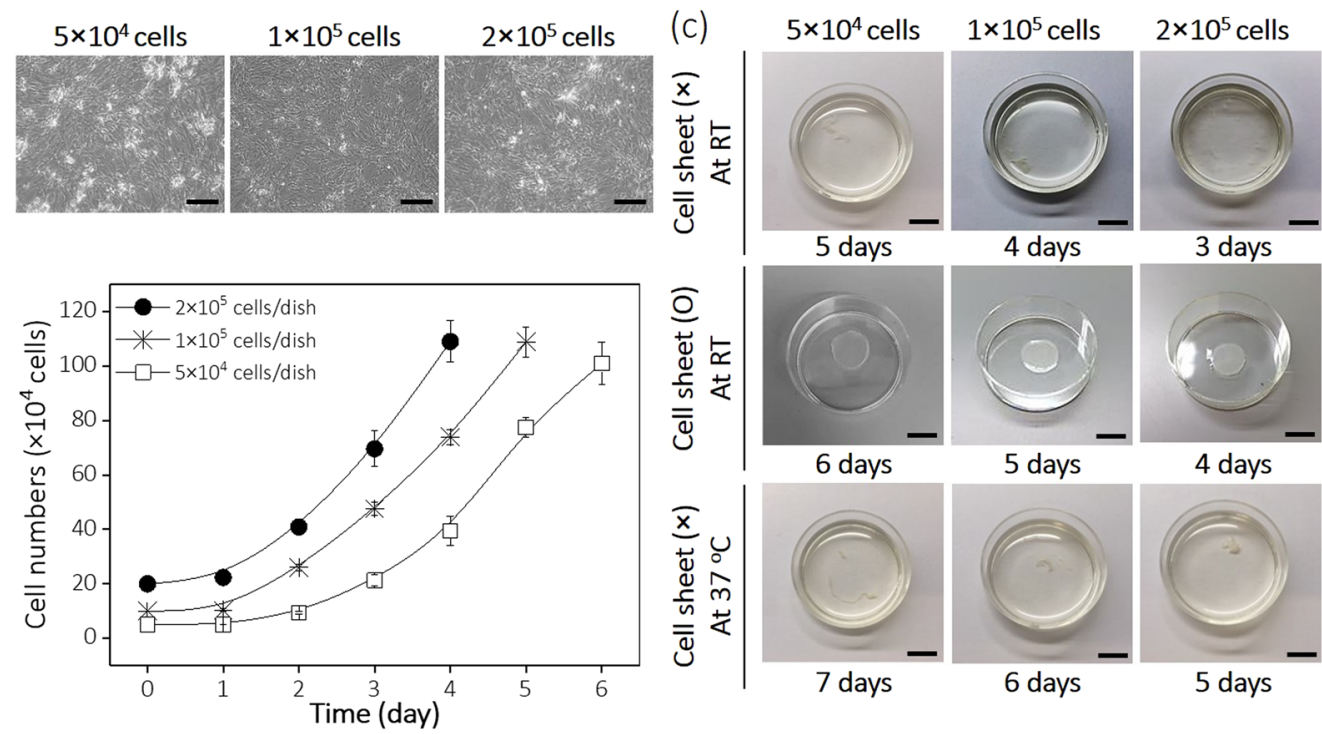

4 days

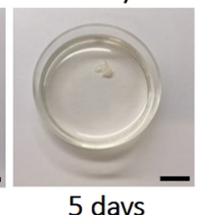

Figure 3. Cell seeding influence. Morphological observation, cell proliferation rate, and cell sheet fabrication for hUC-MSCs seeded at $5 \times 10^{4}, 1 \times 10^{5}$ and $2 \times 10^{5}$ initial cell numbers. (a) Cells cultured on TRCD and observed prior to sheet detachment. (b) Cell numbers counted using a hemacytometer after cells were seeded on TRCD until hUC-MSC sheets are observed. (c) Cells detached as disconnected pieces at one day prior to confluence at 3,4 and 5 days in $2 \times 10^{5}, 1 \times 10^{5}$ and $5 \times 10^{4}$ initial cell seeded groups, respectively. Intact cell sheets were successfully fabricated at 4,5 and 6 day for seeding densities of $2 \times 10^{5}, 1 \times 10^{5}$ and $5 \times 10^{4}$ initial cell number groups, respectively. One day post-confluence, cultured cells spontaneously detach as aggregated forms without TRCD temperature changes at 5, 6 and 7 days for the $2 \times 10^{5}, 1 \times 10^{5}$ and $5 \times 10^{4}$ initial cell seeded groups, respectively. Scale bars indicate $100 \mu \mathrm{m}$ in (a). Scale bars indicate $1 \mathrm{~cm}$ in (c).

(a)
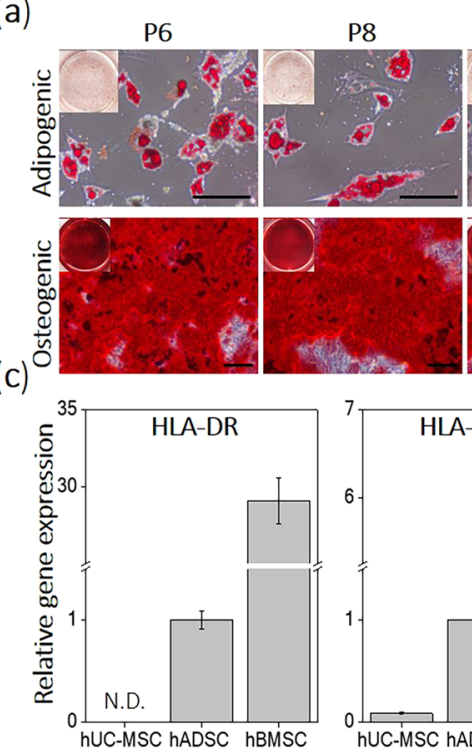

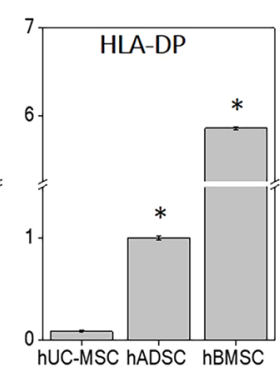

(b)
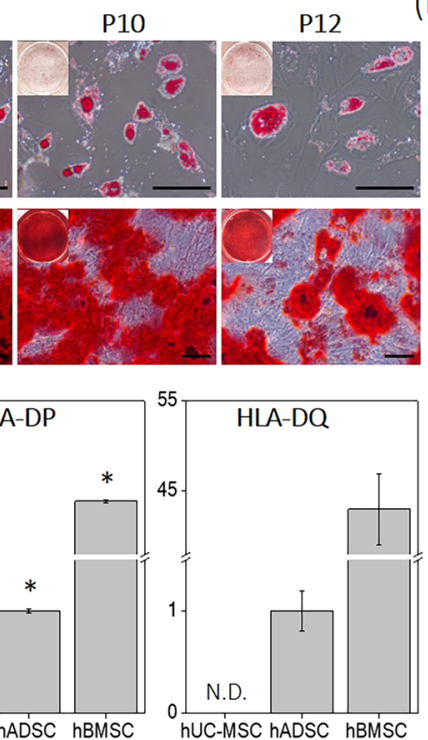

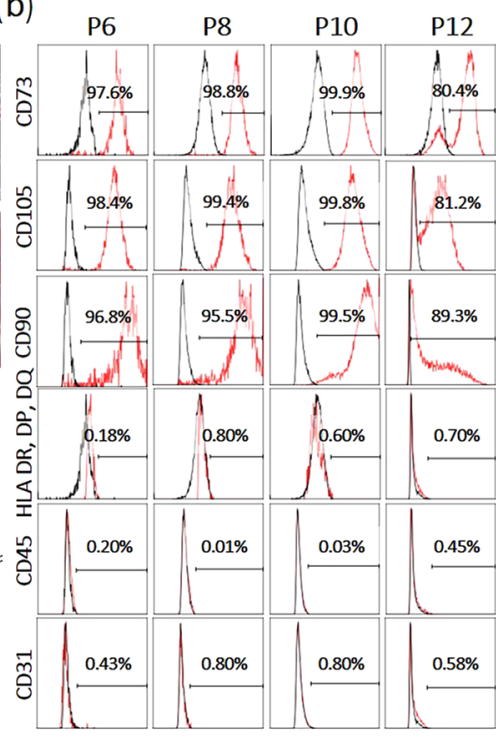

Figure 4. hUC-MSC differentiation potential and stem cell phenotype. hUC-MSCs (passages 6-12) were differentiated into (a) adipocytes and osteocytes. Differentiation potential of hUC-MSCs was dramatically decreased as passage numbers increased. hUC-MSCs displayed positive expression of (b) $\mathrm{CD} 73$ and negative expression of HLA-DR, DP, DQ consistently from passage 6 to 12. (c) HLA-DR, DP, DQ gene expression levels of hUC-MSC sheet were detected by quantitative RT-PCR analysis and compared with other types of MSC sheets (adipose- and bone marrow-derived mesenchymal stem cells) Scale bars in (a) represents $20 \mu \mathrm{m}$. N.D. represents no detection in (c). (c) $* p<0.01$.

phenotypes after sheet detachment. Fibronectin and laminin, important ECM components that promote cell and tissue attachment ${ }^{46-49}$, were strongly expressed across the entire cell sheet surface (Fig. 5b,c) compared to the negative control (Fig. 5a). $\beta$-catenin, part of the protein complex forming cell adherent junctions ${ }^{46}$, shows 


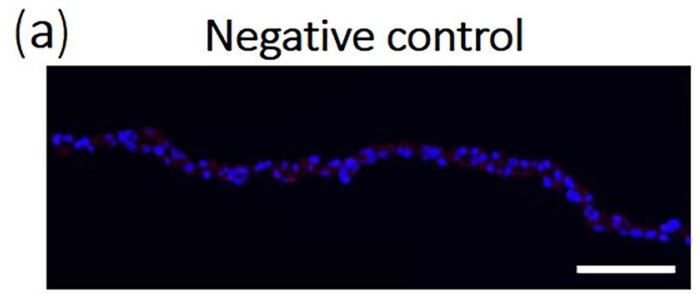

(b)

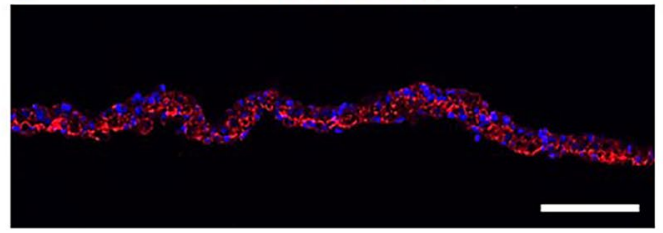

(d)

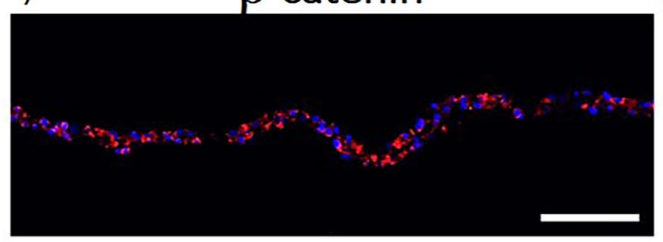

(f)

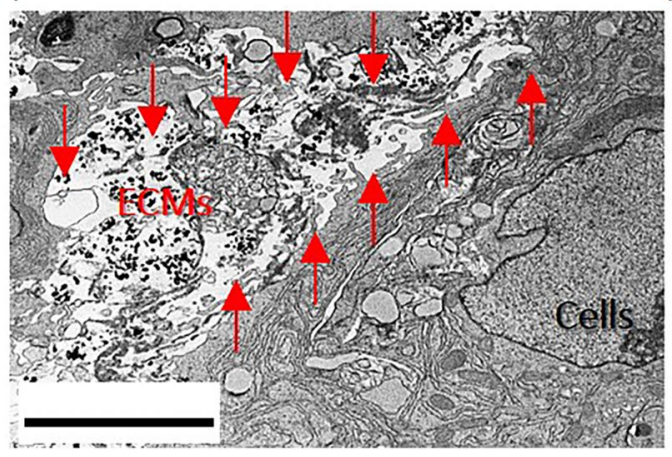

(c)

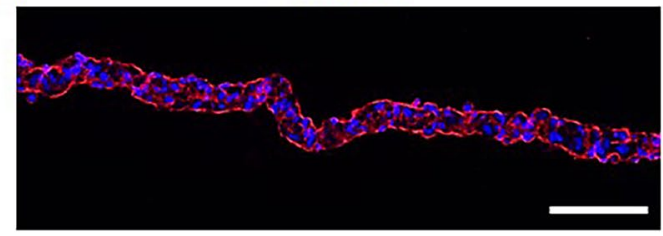

(e)

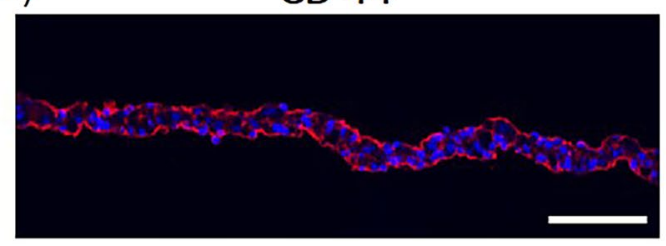

(g)

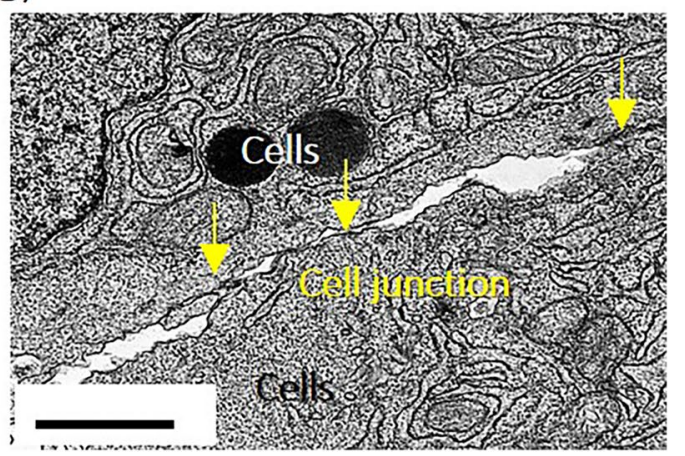

Figure 5. Cell-cell structural analysis using immunohistochemistry (IHC) and transmission electron microscopy (TEM). Cells successfully detached as sheets from TRCD by temperature changes at 4 days after seeding. Cell sheets were stained with isotype negative control antibody (a), ECM ((b) fibronectin and (c) laminin), (d) cell junction $\beta$-catenin, and (e) stem cell surface marker (CD44) antibodies to confirm that cell sheets preserved their functional structures after detachment. In TEM images, hUC-MSC sheets preserved their (e) ECMs and (f) cell-cell junction structures after detachment. Red arrow $=$ ECMs; yellow arrow $=$ cell junctions in the hUC-MSC sheet. Scale bars indicate $100 \mu \mathrm{m}$ in $(\mathbf{a}-\mathbf{d})$. Scale bars $(\mathbf{d}, \mathbf{e})$ indicate $5 \mu \mathrm{m}$ and $1 \mu \mathrm{m}$, respectively.

prominent staining between cells (Fig. 5d). CD44-positive expression, an MSC surface marker ${ }^{50}$, was observed on cell surfaces (Fig. 5e). Retention of ECM, cell junction, and MSC surface proteins indicates that select functional proteins produced during culture are preserved after cell sheet harvest. Inter-cellular structures within cell sheets were observed by TEM. Horizontal sectioning showed ECM structures within cell sheets (Fig. 5f), including numerous cell-cell junctions (Fig. 5g). These results suggest that hUC-MSC sheets structurally retain functional proteins related to natural cell functions such as cell communication and cell adhesion.

Secretion of hepatocyte growth factor (HGF) and tumor necrosis factor-alpha (TNF- $\alpha$ ). Human anti-inflammatory cytokine HGF ${ }^{51,52}$, and pro-inflammatory cytokine TNF- $\alpha^{53}$ secreted from hUC-MSCs in culture supernatant were measured to support paracrine effects of the fabricated hUC-MSC sheets in vitro. No significant differences in amounts of hHGF were seen in $2 \times 10^{5}, 1 \times 10^{5}$, and $5 \times 10^{4}$ cells/dish groups at passage 6 (Fig. 6a). Pro-inflammatory cytokine (hTNF- $\alpha$ ) was barely detectable in $2 \times 10^{5}, 1 \times 10^{5}$, and $5 \times 10^{4}$ cells/dish groups (Fig. 6b). hUC-MSC sheets fabricated using passage 4 cells secreted significantly higher concentrations of hHGF $(633 \mathrm{pg} / \mathrm{mL})$, compared to hUC-MSC sheets fabricated using passage 6, 8, 10, and 12 cells. Amounts of hHGF secreted from hUC-MSC sheets dramatically decreased as passage number increased (Fig. $6 c$ ). hTNF- $\alpha$ 
(a)
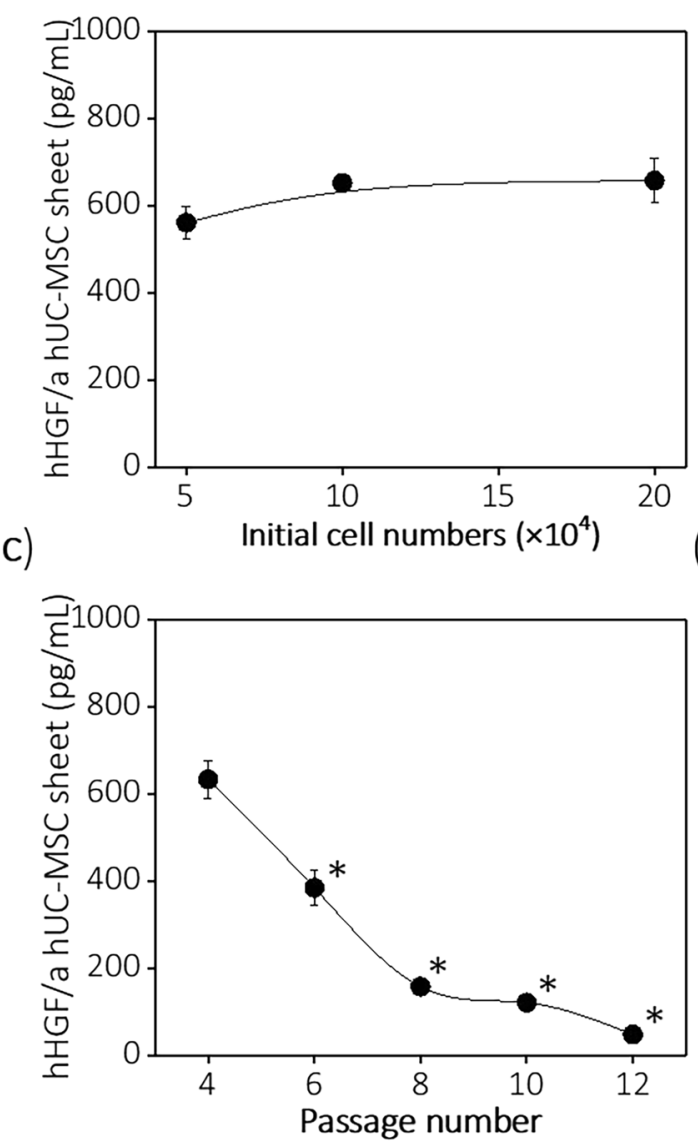

(b)
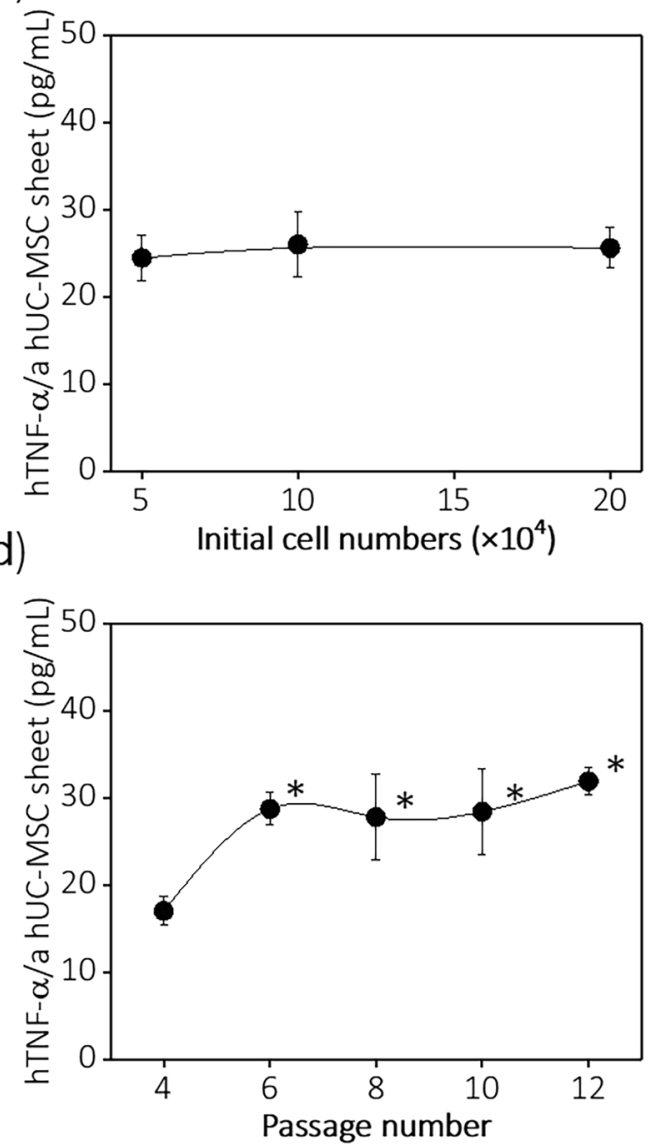

Figure 6. Cytokine analysis of human hepatocyte growth factor (HGF) and tumor necrosis factor-alpha (TNF$\alpha$ ) secreted from hUC-MSC sheets. hHGF (anti-inflammatory cytokine) and hTNF- $\alpha$ (pro-inflammatory cytokine) were detected in culture supernatant for cells cultured for 24 hours. (a) no significant differences in hHGF secretion in $2 \times 10^{5}, 1 \times 10^{5}$, and $5 \times 10^{4}$ initial cell seeded groups. (b) hTNF- $\alpha$ barely detected and not significantly different in $2 \times 10^{5}, 1 \times 10^{5}$ and $5 \times 10^{4}$ initial cell seeded groups. (c) Significant reductions in hHGF secreted from hUC-MSC sheets as passage increases. (d) hUC-MSC sheet fabricated using passage 4 cells secreted significantly lower amount of hTNF- $\alpha$, compared to hUC-MSC sheet fabricated using passage $6,8,10$, and 12 cells $* p<0.05$.

was barely secreted (16-35 pg/mL) from hUC-MSC sheets (Fig. 6d) and hUC-MSC sheets fabricated using passage 4 had significantly lower concentrations of hTNF- $\alpha$, compared to hUC-MSC sheets fabricated using passage $6,8,10$, and 12 cells. Results therefore demonstrate that hUC-MSC passage number is important factor to influence resulting hUC-MSC sheet cytokine secretion properties.

Cell sheet implantation into immune-deficient mice. hUC-MSC sheets were implanted into dorsal subcutaneous pockets in immune-deficient mice for 10 days to demonstrate stability and engraftment in vivo. At 10 days post-transplantation, formation of capillaries (angiogenesis) was observed in cell sheet-transplanted tissue, while subcutaneous tissue without cell sheet transplantation showed only a few fine blood vessels (Fig. 7d,e). H\&E staining data demonstrated that cell sheets remained localized on the transplanted area for 10 days after transplantation (Fig. 7a,b). In cell sheet-transplanted groups, a large number of blood vessel structures was observed between transplanted cell sheets and host tissue (Fig. 7c). Transplanted hUC-MSC sheets secrete human HGF continuously for 10 days in vivo, an important factor for promoting tissue repair (Fig. $7 \mathrm{f}, \mathrm{g})^{54-58}$. This indicates that the hUC-MSC sheets are transplantable and able to secret HGF to target tissue continuously in vivo.

\section{Discussion}

This study describes preparation and properties for hUC-MSC sheets, motivated by the clinical need for new, improved allogeneic MSC cell therapeutic strategies. Harvested using enzymes that compromise MSC functions and engraftment capabilities, current injected MSC cell suspensions exhibit low tissue retention and survival, hence sub-optimal therapeutic properties ${ }^{14}$. Cell sheets created without enzymes and as living constructs bearing native ECM and cell receptors intact can be physically placed onto tissue sites with highly improved retention and engraftment efficiencies ${ }^{32}$. In a previous study, rat adipose- and rat bone marrow- derived MSC sheets 
(a)

w/o cell sheet (X10)

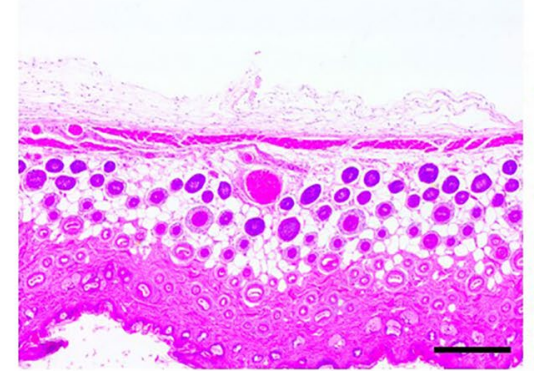

(d)

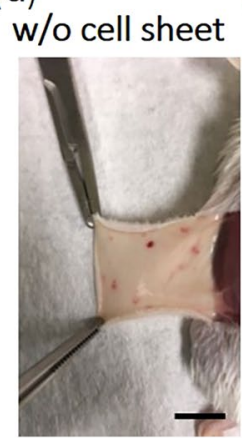

(e)

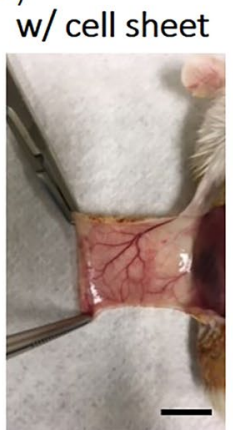

(b)

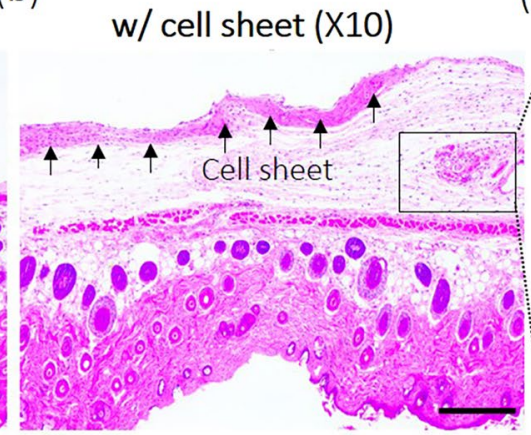

(f) 1 day post transplantation

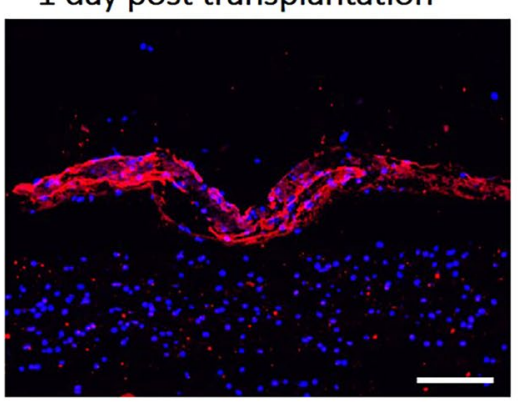

(c)

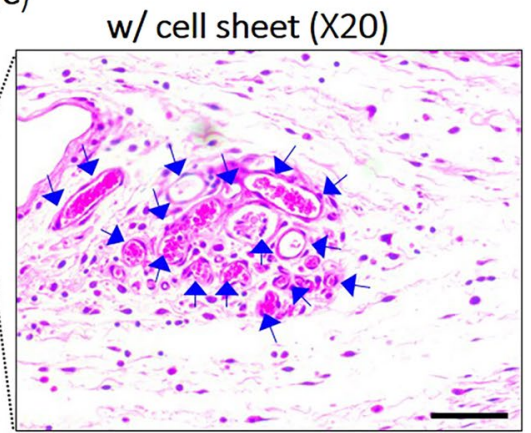

(g)

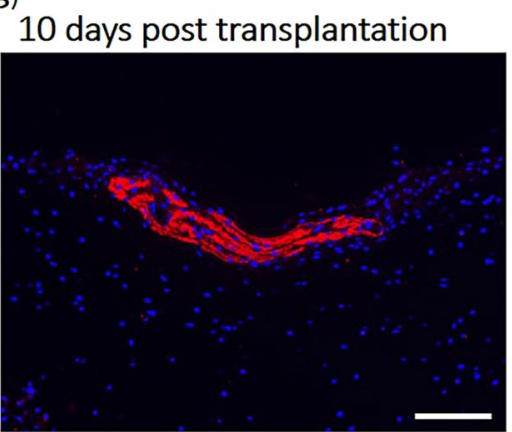

Figure 7. Implanted hUC-MSC sheet retention in vivo. hUC-MSC sheets implanted within subcutaneous tissue in immuno-deficient mice. (d,e) At 10 days after implantation, hUC-MSC transplanted subcutaneous tissue sites were harvested for histological observation. In H\&E-stained images, (b) hu-MSC cell sheets were confirmed clearly in subcutaneous tissue implant sites compared to (a) normal subcutaneous tissue. Additionally, (c) abundant vascular structures are observed in cell sheet implanted groups. hUC-MSC sheets secrete human HGF on the transplanted areas at 1 day (f) and 10 days $(\mathbf{g})$ post implantation. Black arrow $=$ implanted cell sheet; blue arrow $=$ blood vessels. Scale bars $(\mathbf{a}, \mathbf{b})$ and $(\mathbf{c}, \mathbf{f}, \mathbf{g})$ indicate $100 \mu \mathrm{m}, 50 \mu \mathrm{m}$, respectively. Scale bars $(\mathbf{c}, \mathbf{d})$ indicate $0.5 \mathrm{~cm}$.

showed therapeutic effects in repairing scarred myocardium after myocardial infarction ${ }^{30}$, wound healing in a rat model of type 2 diabetes $^{45}$, and in preventing pancreatic leakage ${ }^{44}$. This study creates cell sheets using hUC-MSCs already validated for safety and efficacy in human clinical trials as injected suspensions ${ }^{22-25}$, exhibiting higher paracrine activities with lower allogeneic immune rejection, compared to other MSCs ${ }^{22}$. New MSC sheet fabrication using hUC-MSC can be optimized by exploiting specific culture conditions including passage number, culture time, and initial cell seeding density in this study.

Animal-derived growth supplements, such as fetal bovine serum (FBS), have been widely used for MSC expansion in clinical protocols, and currently accepted by FDA and regulatory bodies for this purpose. However, non-human serum utilized for MSC manufacturing may lead to clinical complications $\mathrm{s}^{59,60}$. Increasingly, xeno-sourced media components are subject to regulatory scrutiny ${ }^{61-63}$. Many approaches have therefore switched MSC culture conditions to human platelet lysate (hPL) media ${ }^{61-63}$. hPL preparations have no standard composition or quality, and diverse sources are reported to increase cell proliferation, differentiation, and immuno-suppressive properties ${ }^{59,64,65}$. Such evidence shows that hPL-containing xeno-free media, while non-standardized, may offer both strategic and performance advantages in clinical-scale MSC expansion towards therapeutic applications. However, cell aggregation using both bone marrow derived (BMSCs) and adipose derived (ADSC) stem cells is reported to occur when cultured in media including $5 \% \mathrm{hPL}^{66,67}$. In Fig. 2, phase contrast microscopy images showed cells stacked on top of each other and formation of cell aggregates at higher passage numbers. This feature tends to increase as passage number increases, especially for passage 12 cells. Active cell adhesive or coagulation factors in hPL could be involved in aggregation, interrupting homogeneous cell growth and cell sheet fabrication processes. To prepare reproducible hUC-MSC sheets, new types of cell culture media to prevent aggregation should be considered.

Central to these results is the reliable capability to produce a stable, robust monolayer of hUC-MSCs using commercial TRCD grafted with temperature-responsive polymer coatings that facilitates cell harvest using temperature reduction without destructive enzymes ${ }^{28,29}$. This cell sheet technology produces cultured cell recovery with intact native cell-cell organization, cell-cell communication, intact ECMs, and tissue-like phenotypes. Cell sheets recovered from TRCDs using small changes in culture temperature preserve cell surface-associated ECMs (e.g., fibronectin and laminin), and cell-cell junction proteins such as $\beta$-catenin (Fig. 5) that play important roles in promoting cell adhesion and paracrine signaling ${ }^{46-49}$. Cell sheets with native morphologies, confluent phenotypes and organization, cell-cell communications, intact extracellular matrix (ECM) and tissue-like behaviors can be readily transferred to target tissues ${ }^{30,32,33}$. Intact ECM in the hUC-MSC sheets served as a natural 
tissue adhesive, producing spontaneous sheet-tissue surface lamination and eliminating needs for sutures on tissue ${ }^{20,30,31}$. hUC-MSC sheets implanted into subcutaneous tissue sites in immune-deficient mice rapidly and spontaneously attached to subcutaneous tissue surfaces within $10 \mathrm{~min}$. After 10 days in vivo, implanted cell sheets remained intact (Fig. 7). MHC class II antigens known to activate the host immune system against allografts ${ }^{68,69}$ must be considered for any off-the-shelf allogenic cell-banked MSC human source. In this regard, hUC-MSC sheets are shown to express considerably low HLA-DR, DP, and DQ (Fig. 4). hUC-MSC sheets fabricated in this study clearly elevate hUC-MSC paracrine benefits without inducing any adverse host inflammatory responses. These new features of hUC-MSC sheets should have considerable value for improving the transplantation efficacy of stem cell therapies.

Tissue regeneration occurs in two contexts: renewal and recruitment of impaired cells to reform damaged organs (structural regeneration), and response to injury by exploiting paracrine and autocrine signals for cell-induced regeneration (functional regeneration $)^{70}$. To regenerate damaged organs, MSCs must be recruited in sufficient numbers and retain a tissue-specific differentiation ability at the target. hUC-MSCs were unable to differentiate sufficiently to chondrocyte phentoypes in Fig. S2. However, hUC-MSCs exhibit both proliferative tendencies and strong differentiation abilities into both adipocyte and osteocyte lineages by passage 10 (Fig. 4). These properties indicate that hUC-MSCs should retain substantial capabilities to replace impaired cells in various tissue sites given proper retention in numbers and phenotypes. Paracrine MSC properties are important in response to injury in tissue regeneration ${ }^{71-74}$. The hUC-MSC is an attractive candidate for regenerative therapy because of its remarkable paracrine action ${ }^{22,26}$. HGF is involved in the mechanism that induces angiogenesis and inhibits fibrosis, and important to tissue repair ${ }^{54-58}$. In a previous study, HGF and VEGF paracrine effects from hUC-MSCs were significantly higher than that of hBMSCs ${ }^{22}$. In this study, after cell sheet fabrication, cell sheets maintained high levels of HGF secretion (Fig. 6). Additionally, passage 4 cells secreted the highest HGF levels in all initial seeding density groups. For disease applications that require paracrine actions to treat, cell sheets fabricated with early passage number cells should prove more effective. Furthermore, human HGF is continuously secreted from implanted hUC-MSC sheets in immune-deficient murine subcutaneous tissue. Neo-vessel formation demonstrated that hUC-MSC sheet implants induced angiogenesis by paracrine action in vivo. hTNF- $\alpha$, a pro-inflmmatory cytokine related to systemic inflammation and hypercalcemia ${ }^{75,76}$ was barely detectable in all groups, including different initial cell seeding and passage numbers.

Overall, hUC-MSC sheets display several beneficial properties for improving allogeneic MSC cell therapy. Results here have determined that (1) specific conditions (cell seeding density, passage number, and culture media) for reliable xeno-free hUC-MSC sheet fabrication are crucial; (2) hUC-MSC sheets preserve important cell functional structures and paracrine effects with low MHC II profiles; (3) hUC-MSC sheet can rapidly adhere to target tissue; and (4) implanted hUC-MSC sheet continually secrete paracrine factors useful to tissue regeneration.

\section{Conclusions}

The simple fabrication method on TRCDs in hPL allows rapid xeno-free production of robust monolayer hUC-MSC sheets, harvested with small temperature changes instead of destructive proteolytic enzymes. Cell sheet production depends on several critical controlled culture variables, including cell seeding density, passage number, and culture time. Given their paracrine effects and low MHC II profile, fabricated xeno-free hUC-MSC sheets represent promising tissue regeneration potential both structurally and functionally in vitro. In future studies, the xenogeneic transplantation of hUC-MSC sheets to wild type mice will provide more information regarding hUC-MSC's immune-modulatory capacity under immune-competent conditions. With reliable topical tissue site placement, long-term retention and survival, and paracrine factor secretion in immune-deficient mice, the hUC-MSC sheet exhibits promising properties for possible future allogeneic cell therapy. However, to enhance reproducibility and simplify the process routine amenable to clinical scaling needs, cell sheet fabrication methods including hPL cell culture media must be further validated. The structure of (double- or triple-) layered cell sheets will be studied as an approach for further enhancing cell sheet function.

\section{Materials and Methods}

Human umbilical cord stem cell (hUC-MSC) culture. Banked human umbilical cord mesenchymal stem cells isolated from the subepithelial layer of human umbilical cord tissue (Jadi Cell LLC, Miami, USA IRB-35242 $)^{27}$ were cultured in xeno-free cell culture media with Dulbecco's Modified Eagle's Medium (DMEM) (Life Technologies, CA, USA) supplemented with 10\% human platelet lysate (hPL, iBiologics, Phoenix, USA), 1\% Glutamax (Life Technologies), 1\% MEM NEAA (Life Technologies), 1\% penicillin streptomycin (Life Technologies), at $37^{\circ} \mathrm{C}$ in a humidified atmosphere with $5 \% \mathrm{CO}_{2}$ for 5 days. The working cell bank was established in passage 4. hUC-MSCs were expanded from passage 4 to 6 to fabricate hUC-MSC sheets. Cell culture media was changed every two days.

hUC-MSC proliferation rates. hUC-MSCs were seeded on 35-mm tissue culture plates (TCP) (Corning, USA) at cell numbers of $5 \times 10^{4}, 1 \times 10^{5}$ and $2 \times 10^{5}$ cells/dish in xeno-free cell culture media. Cells on TCP were dissociated with TrypLE (Gibco, USA) and cell number counted using a hemocytometer at 1, 2, 3, 4, 5 and 6 days. hUC-MSCs were seeded at a cell density of $3.5 \times 10^{3} / \mathrm{cm}^{2}$ on $175 \mathrm{~cm}^{2}$ tissue culture flasks (Corning, USA) and passaged at 5 days with TrypLE after culturing from passage 4 until 12. Cell number was counted each passage using a hemocytometer.

hUC-MSC differentiation potential. hUC-MSCs were cultured in xeno-free cell culture media for two passages on TCP. At passages 6, 8, 10, and 12, cells were prepared and induced for osteogenic and adipogenic 
differentiation. For osteogenic differentiation, cells were plated at $5 \times 10^{3}$ cells $/ \mathrm{cm}^{2}$ in $35 \mathrm{~mm}$ TCP dishes in xeno-free cell culture media. When $60 \%$ confluent, cells were induced with osteogenic differentiation media containing $\alpha \mathrm{MEM}, 10 \mathrm{nM}$ dexamethasone, $82 \mu \mathrm{g} / \mathrm{mL}$ ascorbic acid 2-phosphate, $10 \mathrm{mM} \beta$-glycerolphosphate (Sigma-Aldrich). Cells were cultured in osteogenic media at $37^{\circ} \mathrm{C}$ for 21 days with media changed every 3 days. To detect positive differentiation, cells were fixed with cold $4 \%$ paraformaldehyde (PFA) for 12 minutes and stained with Alizarin Red S- (Sigma-Aldrich) using standard protocols. For adipogenic differentiation, cells were plated at $1 \times 10^{4}$ cells $/ \mathrm{cm}^{2}$ in $35 \mathrm{~mm}$ TCP dishes in xeno-free cell culture media. When $80 \%$ confluent, cells were induced with adipogenic differentiation media containing high-glucose DMEM, $100 \mathrm{nM}$ dexamethasone, $0.5 \mathrm{mM}$ IBMX, and $50 \mu \mathrm{M}$ IND (all Sigma-Aldrich). Cells were cultured in adipogenic media at $37^{\circ} \mathrm{C}$ for 21 days and media changed every 3 days. To detect positive differentiation, cells were fixed with cold $4 \%$ paraformaldehyde for 12 minutes and stained with Oil Red O (Sigma-Aldrich) using standard protocols.

hUC-MSC surface phenotyping assay. hUC-MSCs were cultured in xeno-free cell culture media on TCP. Cells were then detached with TrypLE and washed once with PBS. To minimize non-specific binding of antibodies, cells were incubated with $2 \% \mathrm{w} / \mathrm{v}$ bovine serum albumin (BSA) in PBS for 30 minutes. Cells were then aliquoted at concentrations of $3-5 \times 10^{5} / 100 \mu \mathrm{L}$. One aliquot was reserved as an unstained control and those remaining were stained with the following antibodies: CD73, CD105, CD90, CD45, CD31, and HLA-DR, -DP, -DQ (Biolegend, San Diego, USA). Primary antibody was added to each aliquot to achieve a ratio of about 20:1 of cells in buffer to antibody. About $3-5 \times 10^{5}$ cells were stained with saturating concentrations of (fluorophore)-conjugated antibodies. Cells were incubated in the dark on ice for 30 minutes. After incubation, cells were washed three times and then resuspended in 1X PBS and immediately analyzed by flow cytometry (Becton Dickinson FACS Canto, BD Biosciences, Sparks, USA). Flow cytometer instruments were set using unstained cells. Cells were gated by forward versus side scatter to eliminate doublets. A minimum of 10,000 events was counted for each analysis.

MHC II gene expression tests of cell sheets. Human bone marrow stem cells and adipose-derived stem cells were purchased from Lonza (USA). hUC-MSC, hBMSC and hADSC in passage 6 were seeded at cell numbers of $2 \times 10^{5}$ cells/35-mm dish and cultured for 4-7 days to prepare cell sheets. hUC-MSC cell sheets were collected after detachment from TRCD at RT. Total RNA from cell sheets was extracted using trizol and PureLink RNA Mini Kt (Life Technologies, Carlsbad, USA) according to manufacturer's protocols. cDNA was prepared from 1 $\mu \mathrm{g}$ of total RNA using high capacity cDNA reverse transcription kits (Life Technologies). RT-PCR analysis was performed with TapMan Universal PCR Master Mix using an Applied Biosystems Step One instrument (Applied Biosystems $^{\mathrm{TM}}$, Foster City, USA). Gene expression levels were assessed for the following genes: (1) glyceraldehyde 3-phosphate dehydrogenase (GAPDH, Hs99999905_m1) as a housekeeping gene, (2) human leukocyte antigen (HLA)-DRB (HLA-DR, Hs04192464_m1), (3) HLA-DPB (HLA-DP, Hs03045105_m1), and (4) HLA-DQB (HLA-DQ, Hs03054971_m1). All primers were manufactured by Applied Biosystems (sequences for each shown in Fig. 4). Relative gene expression levels were quantified by the comparative CT method ${ }^{77}$. Gene expression levels were normalized to GAPDH expression levels. Gene expression levels are relative to levels of the hADSC sheet group.

hUC-MSC sheet preparation using different initial cell seeding and passage numbers. hUC-MSC sheets were prepared on TRCDs in various conditions including different initial cell seeding and passage numbers (Fig. 2). Passage 6 cells were seeded on 35-mm TRCDs (Cell Seed Ltd., Tokyo, Japan) at cell numbers of $5 \times 10^{4}$ cells $/$ dish, $1 \times 10^{5}$ cells $/ 35 \mathrm{~mm}$ dish and $2 \times 10^{5}$ cells/dish. Passage $4-12$ cells were seeded at a cell number of $2 \times 10^{5}$ cells/dish. Fresh xeno-free cell culture media including $16.4 \mu \mathrm{g} / \mathrm{mL}$ of ascorbic acid (Sigma-Aldrich, St. Louis, USA) to make cell sheets was added at 1 day after seeding. Confluent cell sheets formed 4-6 days after seeding and were detached from TRCD at RT (Fig. 1). Cell morphologies were monitored using an AX10 microscope (Carl Zeiss Microimaging, Göttingen, Germany) with AxioVision software (Carl Zeiss Microimaging) before cell sheet detachment.

Histological analysis. Cultured passage 6 cell sheets were removed from TRCD at RT and fixed with 4\% PFA for $30 \mathrm{~min}$ and then embedded in paraffin. Embedded specimens were sectioned into $4 \mu \mathrm{m}$ slices and stained with H\&E, isotype immunoglobulin G (IgG) antibody (negative control), stem cell surface markers (CD44), ECM proteins (fibronectin; FN and laminin; LM) and cell-cell junctions (integrin-linked kinase; $\beta$-catenin). For fluorescence staining (IgG, CD44, FN, LM, and $\beta$-catenin), slides were immersed in antigen retriever solution (Sigma-Aldrich) for $20 \mathrm{~min}$ at $100^{\circ} \mathrm{C}$ and washed with PBS 1X. Non-specific binding was blocked in PBS 1X containing 10\% goat serum (Vector Laboratories, Burlingame, USA), for $1 \mathrm{~h}$ at room temperature. Primary antibody labeling (Abcam, Cambridge, USA) $(1: 100)$ at $4{ }^{\circ} \mathrm{C}$ proceeded overnight and then washed with PBS $1 \mathrm{X}$. These specimens were treated with Alexa Fluor 594-conjugated secondary antibodies (Life Technologies) (1:200) for $1 \mathrm{~h}$ and mounted with ProLong Gold Antifade Reagent (Life Technologies). Immunofluorescence images were obtained using an AX 10 microscope (Carl Zeiss Microimaging) and analyzed with Axiovision software (Carl Zeiss Microimaging). For H\&E staining, specimens were treated with hematoxylin solution (Sigma-Aldrich) for $3 \mathrm{~min}$ and subsequently with eosin solution (ThermoFisher Scientific, Kalamazoo, USA) for 5 min. The H\&E stained specimens were dehydrated and mounted with Permount ${ }^{\mathrm{TM}}$ (ThermoFisher Scientific, USA). H\&E images were obtained using a BX 41 microscope (Olympus, Hamburg, Germany).

Cell sheet microstructure observed using transmission electron microscopy. hUC-MSC sheets at passage 6 were fixed with a mixture of $2 \%$ paraformaldehyde, $2 \%$ glutaraldehyde, $0.1 \mathrm{M}$ sodium phosphate buffer, and $2 \%$ osmium tetroxide $\left(\mathrm{OsO}_{4}\right)$ in sodium phosphate buffer and dehydrated in a grade series of ethanol. 
Samples were then embedded in epoxy resin. Ultrathin sections ( $70 \mathrm{~nm}$ thickness) were observed with a transmission electron microscope (JEOL JEM1200EX, JEOL USA, Peabody, USA).

Determination of hepatocyte growth factor (HGF) and tumor necrosis factor alpha (TNF- $\alpha$ ) secretion from hUC-MSC sheets. hUC-MSC cell sheets at passage 6 were fabricated on TRCDs. Supernatant media over adherent cultured cells for 24 hours was collected just prior to cell sheet detachment from TRCD at RT. HGF and TNF- $\alpha$ amounts secreted from hUC-MSCs were measured by human HGF Quantikine ELISA and human TNF- $\alpha$ Quantikine ELISA kits, respectively (R\&D Systems, Minneapolis, USA).

Cell sheet placement into immune-deficient mice subcutaneous tissue. Six-week old immune-deficient mice (NOD.CB17-Prkdc ${ }^{\text {scid}} / \mathrm{NCrCrl}$ ) (Charles River, San Diego, USA) were anesthetized by inhalation of $5 \%$ isoflurane. The percent isoflurane was decreased to $1-3 \%$ once the rodent was fully sedated. One small incision (approx. $3 \mathrm{~cm}$ ) was made approximately near the center of the back and a standard subcutaneous dorsal pocket was generated using blunt dissection ${ }^{78}$. hUC-MSC (passage 6) cell sheets ( $1 \mathrm{~cm}$ diameter circles) were detached from TRCD at RT after 4 days of culture and transplanted into subcutaneous dorsal tissue pockets ${ }^{78}$. Sterilized non-cytotoxic silicone membranes (a rectangle with length $1.2 \mathrm{~cm}$, width $1.2 \mathrm{~cm}$, and $0.3 \mathrm{~mm}$ thickness) (Invitrogen) were placed between each implanted cell sheet and subcutaneous dorsal tissues to block tissue adhesion. The incision was closed with a simple interrupted pattern using absorbable thread. Implanted mice $(n=6)$ were sacrificed at 10 days after cell sheet transplantation. The cell sheet-transplanted subcutaneous tissue was harvested and fixed with $10 \%$ formaldehyde (Sigma-Aldrich) for 1 day for histological analysis (see Materials and Methods; 2.7. Histological analysis). All procedures were approved by the Institutional Animal Care and Use Committee (IACUC) (protocol \#16-12017) at The University of Utah and conducted in accordance with accepted international guidelines.

Statistical analyses. All quantitative values are expressed as mean and standard error (SE, mean $\pm \mathrm{SE}$ ). Significant differences between groups were tested by one-way Analysis of Variance using Origin 2017 software (OriginLab, Northampton, USA). A probability value of less than $0.05(p<0.05)$ was considered statistically significant.

\section{References}

1. Galipeau, J. \& Sensebe, L. Mesenchymal Stromal Cells: Clinical Challenges and Therapeutic Opportunities. Cell Stem Cell 22, 824-833, https://doi.org/10.1016/j.stem.2018.05.004 (2018).

2. Squillaro, T., Peluso, G. \& Galderisi, U. Clinical Trials With Mesenchymal Stem Cells: An Update. Cell Transplant 25, 829-848, https://doi.org/10.3727/096368915X689622 (2016).

3. Wei, X. et al. Mesenchymal stem cells: a new trend for cell therapy. Acta Pharmacol Sin 34, 747-754, https://doi.org/10.1038/ aps.2013.50 (2013)

4. Potian, J. A., Aviv, H., Ponzio, N. M., Harrison, J. S. \& Rameshwar, P. Veto-like activity of mesenchymal stem cells: functional discrimination between cellular responses to alloantigens and recall antigens. J Immunol 171, 3426-3434 (2003).

5. Le Blanc, K., Tammik, C., Rosendahl, K., Zetterberg, E. \& Ringden, O. HLA expression and immunologic properties of differentiated and undifferentiated mesenchymal stem cells. Exp Hematol 31, 890-896 (2003).

6. Krampera, M. et al. Bone marrow mesenchymal stem cells inhibit the response of naive and memory antigen-specific $\mathrm{T}$ cells to their cognate peptide. Blood 101, 3722-3729, https://doi.org/10.1182/blood-2002-07-2104 (2003).

7. Ryan, J. M., Barry, F. P., Murphy, J. M. \& Mahon, B. P. Mesenchymal stem cells avoid allogeneic rejection. J Inflamm (Lond) 2 , 8, https://doi.org/10.1186/1476-9255-2-8 (2005).

8. Jiang, X. X. et al. Human mesenchymal stem cells inhibit differentiation and function of monocyte-derived dendritic cells. Blood 105, 4120-4126, https://doi.org/10.1182/blood-2004-02-0586 (2005).

9. Shake, J. G. et al. Mesenchymal stem cell implantation in a swine myocardial infarct model: engraftment and functional effects. Ann Thorac Surg 73, 1919-1925; discussion 1926 (2002).

10. Murphy, J. M., Fink, D. J., Hunziker, E. B. \& Barry, F. P. Stem cell therapy in a caprine model of osteoarthritis. Arthritis Rheum 48, 3464-3474, https://doi.org/10.1002/art.11365 (2003).

11. Keilhoff, G., Stang, F., Goihl, A., Wolf, G. \& Fansa, H. Transdifferentiated mesenchymal stem cells as alternative therapy in supporting nerve regeneration and myelination. Cell Mol Neurobiol 26, 1235-1252, https://doi.org/10.1007/s10571-006-9029-9 (2006).

12. Abou-El-Enein, M., Elsanhoury, A. \& Reinke, P. Overcoming Challenges Facing Advanced Therapies in the EU Market. Cell Stem Cell 19, 293-297, https://doi.org/10.1016/j.stem.2016.08.012 (2016).

13. Leor, J. et al. Bioengineered cardiac grafts: A new approach to repair the infarcted myocardium? Circulation 102, III56-61 (2000).

14. Devine, S. M., Cobbs, C., Jennings, M., Bartholomew, A. \& Hoffman, R. Mesenchymal stem cells distribute to a wide range of tissues following systemic infusion into nonhuman primates. Blood 101, 2999-3001, https://doi.org/10.1182/blood-2002-06-1830 (2003).

15. Reinecke, H. \& Murry, C. E. Taking the death toll after cardiomyocyte grafting: a reminder of the importance of quantitative biology. J Mol Cell Cardiol 34, 251-253, https://doi.org/10.1006/jmcc.2001.1494 (2002).

16. Reiser, J. et al. Potential of mesenchymal stem cells in gene therapy approaches for inherited and acquired diseases. Expert Opin Biol Ther 5, 1571-1584, https://doi.org/10.1517/14712598.5.12.1571 (2005).

17. Baxter, M. A. et al. Study of telomere length reveals rapid aging of human marrow stromal cells following in vitro expansion. Stem Cells 22, 675-682, https://doi.org/10.1634/stemcells.22-5-675 (2004).

18. Banfi, A. et al. Proliferation kinetics and differentiation potential of ex vivo expanded human bone marrow stromal cells: Implications for their use in cell therapy. Exp Hematol 28, 707-715 (2000).

19. Ikebe, C. \& Suzuki, K. Mesenchymal stem cells for regenerative therapy: optimization of cell preparation protocols. Biomed Res Int 2014, 951512, https://doi.org/10.1155/2014/951512 (2014).

20. Yamato, M. et al. Thermo-responsive culture dishes allow the intact harvest of multilayered keratinocyte sheets without dispase by reducing temperature. Tissue Eng 7, 473-480, https://doi.org/10.1089/10763270152436517 (2001).

21. Terrovitis, J. V., Smith, R. R. \& Marban, E. Assessment and optimization of cell engraftment after transplantation into the heart. Circ Res 106, 479-494, https://doi.org/10.1161/CIRCRESAHA.109.208991 (2010).

22. Bartolucci, J. et al. Safety and Efficacy of the Intravenous Infusion of Umbilical Cord Mesenchymal Stem Cells in Patients With Heart Failure: A Phase 1/2 Randomized Controlled Trial (RIMECARD Trial [Randomized Clinical Trial of Intravenous Infusion Umbilical Cord Mesenchymal Stem Cells on Cardiopathy]). Circ Res 121, 1192-1204, https://doi.org/10.1161/CIRCRESAHA.117.310712 (2017). 
23. Ichim, T. E. et al. Feasibility of combination allogeneic stem cell therapy for spinal cord injury: a case report. Int Arch Med 3, 30, https://doi.org/10.1186/1755-7682-3-30 (2010).

24. Riordan, N. H. et al. Clinical feasibility of umbilical cord tissue-derived mesenchymal stem cells in the treatment of multiple sclerosis. J Transl Med 16, 57, https://doi.org/10.1186/s12967-018-1433-7 (2018).

25. Tuma, J. et al. RESCUE-HF Trial: Retrograde Delivery of Allogeneic Umbilical Cord Lining Subepithelial Cells in Patients With Heart Failure. Cell Transplant 25, 1713-1721, https://doi.org/10.3727/096368915X690314 (2016).

26. Winters, A. A. et al. Evaluation of Multiple Biological Therapies for Ischemic Cardiac Disease. Cell Transplant 25, 1591-1607, https:// doi.org/10.3727/096368916X691501 (2016).

27. Patel, A. N., Vargas, V., Revello, P. \& Bull, D. A. Mesenchymal stem cell population isolated from the subepithelial layer of umbilical cord tissue. Cell Transplant 22, 513-519, https://doi.org/10.3727/096368912X655064 (2013).

28. Okano, T., Yamada, N., Okuhara, M., Sakai, H. \& Sakurai, Y. Mechanism of cell detachment from temperature-modulated, hydrophilic-hydrophobic polymer surfaces. Biomaterials 16, 297-303 (1995).

29. Okano, T., Yamada, N., Sakai, H. \& Sakurai, Y. A novel recovery system for cultured cells using plasma-treated polystyrene dishes grafted with poly(N-isopropylacrylamide). J Biomed Mater Res 27, 1243-1251, https://doi.org/10.1002/jbm.820271005 (1993).

30. Miyahara, Y. et al. Monolayered mesenchymal stem cells repair scarred myocardium after myocardial infarction. Nat Med 12, 459-465, https://doi.org/10.1038/nm1391 (2006).

31. Kushida, A. et al. Decrease in culture temperature releases monolayer endothelial cell sheets together with deposited fibronectin matrix from temperature-responsive culture surfaces. J Biomed Mater Res 45, 355-362 (1999).

32. Sekine, H. et al. Cardiac cell sheet transplantation improves damaged heart function via superior cell survival in comparison with dissociated cell injection. Tissue Eng Part A 17, 2973-2980, https://doi.org/10.1089/ten.tea.2010.0659 (2011).

33. Nishida, K. et al. Corneal reconstruction with tissue-engineered cell sheets composed of autologous oral mucosal epithelium. $N$ Engl J Med 351, 1187-1196, https://doi.org/10.1056/NEJMoa040455 (2004).

34. Ebihara, G. et al. Cartilage repair in transplanted scaffold-free chondrocyte sheets using a minipig model. Biomaterials 33, 3846-3851, https://doi.org/10.1016/j.biomaterials.2012.01.056 (2012).

35. Iwata, T. et al. Cell Sheets for Periodontal Tissue Engineering. Current Oral Health Reports 2, 252-256, https://doi.org/10.1007/ s40496-015-0063-x (2015).

36. Iwata, T. et al. Periodontal regeneration with autologous periodontal ligament-derived cell sheets - A safety and efficacy study in ten patients. Regenerative Therapy 9, 38-44, https://doi.org/10.1016/j.reth.2018.07.002 (2018).

37. Kanzaki, M., Takagi, R., Washio, K., Kokubo, M. \& Yamato, M. Bio-artificial pleura using an autologous dermal fibroblast sheet. NPJ Regen Med 2, 26, https://doi.org/10.1038/s41536-017-0031-2 (2017).

38. Nishida, K. et al. Functional bioengineered corneal epithelial sheet grafts from corneal stem cells expanded ex vivo on a temperatureresponsive cell culture surface. Transplantation 77, 379-385, https://doi.org/10.1097/01.TP.0000110320.45678.30 (2004).

39. Ohki, T. et al. Prevention of esophageal stricture after endoscopic submucosal dissection using tissue-engineered cell sheets. Gastroenterology 143, 582-588 e582, https://doi.org/10.1053/j.gastro.2012.04.050 (2012).

40. Sawa, Y. et al. Tissue engineered myoblast sheets improved cardiac function sufficiently to discontinue LVAS in a patient with DCM: report of a case. Surg Today 42, 181-184, https://doi.org/10.1007/s00595-011-0106-4 (2012).

41. Sawa, Y. et al. Safety and Efficacy of Autologous Skeletal Myoblast Sheets (TCD-51073) for the Treatment of Severe Chronic Heart Failure Due to Ischemic Heart Disease. Circ J 79, 991-999, https://doi.org/10.1253/circj.CJ-15-0243 (2015).

42. Yamaguchi, N. et al. Oral epithelial cell sheets engraftment for esophageal strictures after endoscopic submucosal dissection of squamous cell carcinoma and airplane transportation. Sci Rep 7, 17460, https://doi.org/10.1038/s41598-017-17663-w (2017).

43. Yamamoto, K. et al. Middle ear mucosal regeneration by tissue-engineered cell sheet transplantation. NPJ Regen Med 2, 6, https:// doi.org/10.1038/s41536-017-0010-7 (2017).

44. Kim, S. R. et al. Engineered mesenchymal stem-cell-sheets patches prevents postoperative pancreatic leakage in a rat model. Sci Rep 8, 360, https://doi.org/10.1038/s41598-017-18490-9 (2018).

45. Kato, Y. et al. Allogeneic Transplantation of an Adipose-Derived Stem Cell Sheet Combined With Artificial Skin Accelerates Wound Healing in a Rat Wound Model of Type 2 Diabetes and Obesity. Diabetes 64, 2723-2734, https://doi.org/10.2337/db14-1133 (2015).

46. Nelson, W. J. \& Nusse, R. Convergence of Wnt, beta-catenin, and cadherin pathways. Science 303, 1483-1487, https://doi. org/10.1126/science.1094291 (2004).

47. Frantz, C., Stewart, K. M. \& Weaver, V. M. The extracellular matrix at a glance. J Cell Sci 123, 4195-4200, https://doi.org/10.1242/ jcs.023820 (2010).

48. Gattazzo, F., Urciuolo, A. \& Bonaldo, P. Extracellular matrix: a dynamic microenvironment for stem cell niche. Biochim Biophys Acta 1840, 2506-2519, https://doi.org/10.1016/j.bbagen.2014.01.010 (2014)

49. Brownlee, C. Role of the extracellular matrix in cell-cell signalling: paracrine paradigms. Curr Opin Plant Biol 5, 396-401 (2002).

50. Lv, F. J., Tuan, R. S., Cheung, K. M. \& Leung, V. Y. Concise review: the surface markers and identity of human mesenchymal stem cells. Stem Cells 32, 1408-1419, https://doi.org/10.1002/stem.1681 (2014).

51. Zhang, J., Middleton, K. K., Fu, F. H., Im, H. J. \& Wang, J. H. HGF mediates the anti-inflammatory effects of PRP on injured tendons. PLoS One 8, e67303, https://doi.org/10.1371/journal.pone.0067303 (2013).

52. Gong, R., Rifai, A. \& Dworkin, L. D. Anti-inflammatory effect of hepatocyte growth factor in chronic kidney disease: targeting the inflamed vascular endothelium. J Am Soc Nephrol 17, 2464-2473, https://doi.org/10.1681/ASN.2006020185 (2006).

53. Ertel, W. et al. Downregulation of proinflammatory cytokine release in whole blood from septic patients. Blood 85, 1341-1347 (1995).

54. Inoue, T. et al. Hepatocyte growth factor counteracts transforming growth factor-beta1, through attenuation of connective tissue growth factor induction, and prevents renal fibrogenesis in 5/6 nephrectomized mice. FASEB J 17, 268-270, https://doi.org/10.1096/ f. $02-0442$ fje (2003).

55. Mizuno, S., Matsumoto, K., Li, M. Y. \& Nakamura, T. HGF reduces advancing lung fibrosis in mice: a potential role for MMPdependent myofibroblast apoptosis. FASEB J 19, 580-582, https://doi.org/10.1096/fj.04-1535fje (2005).

56. Mungunsukh, O., McCart, E. A. \& Day, R. M. Hepatocyte Growth Factor Isoforms in Tissue Repair, Cancer, and Fibrotic Remodeling. Biomedicines 2, 301-326, https://doi.org/10.3390/biomedicines2040301 (2014).

57. Neuss, S., Becher, E., Woltje, M., Tietze, L. \& Jahnen-Dechent, W. Functional expression of HGF and HGF receptor/c-met in adult human mesenchymal stem cells suggests a role in cell mobilization, tissue repair, and wound healing. Stem Cells 22, 405-414, https:// doi.org/10.1634/stemcells.22-3-405 (2004).

58. Taniyama, Y. et al. Angiogenesis and antifibrotic action by hepatocyte growth factor in cardiomyopathy. Hypertension 40, 47-53 (2002).

59. Oikonomopoulos, A. et al. Optimization of human mesenchymal stem cell manufacturing: the effects of animal/xeno-free media. Sci Rep 5, 16570, https://doi.org/10.1038/srep16570 (2015).

60. Halme, D. G. \& Kessler, D. A. FDA regulation of stem-cell-based therapies. N Engl J Med 355, 1730-1735, https://doi.org/10.1056/ NEJMhpr063086 (2006).

61. Shih, D. T. \& Burnouf, T. Preparation, quality criteria, and properties of human blood platelet lysate supplements for ex vivo stem cell expansion. N Biotechnol 32, 199-211, https://doi.org/10.1016/j.nbt.2014.06.001 (2015).

62. Burnouf, T., Strunk, D., Koh, M. B. \& Schallmoser, K. Human platelet lysate: Replacing fetal bovine serum as a gold standard for human cell propagation? Biomaterials 76, 371-387, https://doi.org/10.1016/j.biomaterials.2015.10.065 (2016). 
63. Astori, G. et al. Platelet lysate as a substitute for animal serum for the ex-vivo expansion of mesenchymal stem/stromal cells: present and future. Stem Cell Res Ther 7, 93, https://doi.org/10.1186/s13287-016-0352-x (2016).

64. Bieback, K. et al. Human alternatives to fetal bovine serum for the expansion of mesenchymal stromal cells from bone marrow. Stem Cells 27, 2331-2341, https://doi.org/10.1002/stem.139 (2009).

65. Lohmann, M. et al. Donor age of human platelet lysate affects proliferation and differentiation of mesenchymal stem cells. PLoS One 7, e37839, https://doi.org/10.1371/journal.pone.0037839 (2012).

66. Juhl, M. et al. Comparison of clinical grade human platelet lysates for cultivation of mesenchymal stromal cells from bone marrow and adipose tissue. Scand J Clin Lab Invest 76, 93-104, https://doi.org/10.3109/00365513.2015.1099723 (2016).

67. Hemeda, H., Giebel, B. \& Wagner, W. Evaluation of human platelet lysate versus fetal bovine serum for culture of mesenchymal stromal cells. Cytotherapy 16, 170-180, https://doi.org/10.1016/j.jcyt.2013.11.004 (2014).

68. Zantvoort, F. A. et al. The impact of HLA-A matching on long-term survival of renal allografts. Transplantation 61, 841-844 (1996).

69. Takemoto, S. K., Terasaki, P. I., Gjertson, D. W. \& Cecka, J. M. Twelve years' experience with national sharing of HLA-matched cadaveric kidneys for transplantation. N Engl J Med 343, 1078-1084, https://doi.org/10.1056/NEJM200010123431504 (2000).

70. Bergmann, A. \& Steller, H. Apoptosis, stem cells, and tissue regeneration. Sci Signal 3, re8, https://doi.org/10.1126/scisignal.3145re8 (2010).

71. Caplan, A. I. Adult mesenchymal stem cells for tissue engineering versus regenerative medicine. J Cell Physiol 213, 341-347, https:// doi.org/10.1002/jcp.21200 (2007).

72. Caplan, A. I. \& Correa, D. The MSC: an injury drugstore. Cell Stem Cell 9, 11-15, https://doi.org/10.1016/j.stem.2011.06.008 (2011).

73. Kusuma, G. D., Carthew, J., Lim, R. \& Frith, J. E. Effect of the Microenvironment on Mesenchymal Stem Cell Paracrine Signaling: Opportunities to Engineer the Therapeutic Effect. Stem Cells Dev 26, 617-631, https://doi.org/10.1089/scd.2016.0349 (2017).

74. Park, C. W. et al. Cytokine secretion profiling of human mesenchymal stem cells by antibody array. Int I Stem Cells 2, 59-68 (2009).

75. Beutler, B. \& Cerami, A. The biology of cachectin/TNF-a primary mediator of the host response. Annu Rev Immunol 7, 625-655, https://doi.org/10.1146/annurev.iy.07.040189.003205 (1989).

76. Klein, J. \& Sato, A. The HLA system. Second of two parts. N Engl J Med 343, 782-786, https://doi.org/10.1056/NEJM200009143431106 (2000).

77. Schmittgen, T. D. \& Livak, K. J. Analyzing real-time PCR data by the comparative C(T) method. Nat Protoc 3, 1101-1108 (2008).

78. Tatsumi, K. et al. A novel cell-sheet technology that achieves durable factor VIII delivery in a mouse model of hemophilia A. PLoS One 8, e83280, https://doi.org/10.1371/journal.pone.0083280 (2013).

\section{Acknowledgements}

This work was supported by University of Utah Health Sciences translational research partnerships, and the University Technology Acceleration Grant from Utah Science, Technology, and Research (USTAR) program, Utah, USA. hUC-MSCs were kindly provided by Jadi Cell LLC, Miami, USA. We thank Dr. Amit N. Patel (University of Miami, USA) for scientific advice about hUC-MSC culture and clinical utility.

\section{Author Contributions}

K.K. and T.O. conceived the idea of the study, designed the experiments and supervised the project. K.K. wrote the main manuscript text and prepared Figures 1-3, 4c and 5-7, and supplemental figure 1 and 3. S.B. prepared Figure $4 \mathrm{~b}$ and H.T. prepared Figure $4 \mathrm{a}$ and supplemental figure 2. T.O. and D.G. reviewed, suggested technical improvements, and edited the manuscript.

\section{Additional Information}

Supplementary information accompanies this paper at https://doi.org/10.1038/s41598-019-50430-7.

Competing Interests: Teruo Okano holds equity in CellSeed, Inc. (Japan) and is an inventor/developer designated on patents for CellSeed's commercialized temperature-responsive culture surfaces. No other competing financial interests exist. The authors declare that they have no competing interests.

Publisher's note Springer Nature remains neutral with regard to jurisdictional claims in published maps and institutional affiliations.

(c) (i) Open Access This article is licensed under a Creative Commons Attribution 4.0 International cc) License, which permits use, sharing, adaptation, distribution and reproduction in any medium or format, as long as you give appropriate credit to the original author(s) and the source, provide a link to the Creative Commons license, and indicate if changes were made. The images or other third party material in this article are included in the article's Creative Commons license, unless indicated otherwise in a credit line to the material. If material is not included in the article's Creative Commons license and your intended use is not permitted by statutory regulation or exceeds the permitted use, you will need to obtain permission directly from the copyright holder. To view a copy of this license, visit http://creativecommons.org/licenses/by/4.0/.

(C) The Author(s) 2019 